Comment. Math. Helv. 75 (2000) 430-456

(C) 2000 Birkhäuser Verlag, Basel

$0010-2571 / 00 / 030430-27 \$ 1.50+0.20 / 0$

Commentarii Mathematici Helvetici

\title{
Annular Dehn fillings
}

\author{
Cameron McA. Gordon ${ }^{1}$ and Ying-Qing $\mathrm{Wu}^{2}$
}

\begin{abstract}
We show that if a simple 3-manifold $M$ has two Dehn fillings at distance $\Delta \geq 4$, each of which contains an essential annulus, then $M$ is one of three specific 2-component link exteriors in $S^{3}$. One of these has such a pair of annular fillings with $\Delta=5$, and the other two have pairs with $\Delta=4$.
\end{abstract}

Mathematics Subject Classification (2000). Primary 57N10.

Keywords. 3-manifolds, Dehn filling, essential annuli.

\section{$\S 1$. Introduction}

Let $M$ be a (compact, connected, orientable) 3-manifold with a torus boundary component $T_{0}$. If $r$ is a slope (the isotopy class of an essential unoriented simple loop) on $T_{0}$, then as usual we denote by $M(r)$ the 3 -manifold obtained from $M$ by $r$-Dehn filling, that is, attaching a solid torus $J$ to $M$ along $T_{0}$ in such a way that $r$ bounds a meridian disk in $J$.

We shall say that a compact, connected, orientable 3-manifold $M$ is simple if it contains no essential surface of non-negative Euler characteristic, i.e., sphere, disk, annulus or torus. If $M$ has non-empty boundary and is not the 3 -ball, then $M$ is simple if and only if $M$ with its boundary tori removed has a complete hyperbolic structure of finite volume with totally geodesic boundary [Th1, Th2]. If $M$ is closed, then the geometrization conjecture asserts that $M$ is simple if and only if $M$ is either hyperbolic or belongs to a certain small class of Seifert fiber spaces [Th1, Th2].

If $M$ is hyperbolic, then Dehn fillings on $M$ are hyperbolic if we exclude finitely many slopes from each torus boundary component [Th1, Th2]. By doubling $M$ along its non-torus boundary components, we see that if $M$ is simple then $M(r)$ is simple for all but finitely many slopes $r$ on any given torus boundary component $T_{0}$, and a good deal of attention has been directed towards obtaining a more precise

\footnotetext{
${ }^{1}$ Partially supported by NSF grant \#DMS 9626550.

2 Partially supported by NSF grant \#DMS 9802558.
} 
quantification of this statement. Denote by $\Delta\left(r_{1}, r_{2}\right)$ the distance, or minimal geometric intersection number, between two slopes $r_{1}, r_{2}$ on a torus. Define a 3-manifold to be of type $S, D, A$ or $T$ if it contains an essential sphere, disk, annulus or torus, respectively. For $X_{i} \in\{S, D, A, T\}, i=1,2$, define $\Delta\left(X_{1}, X_{2}\right)$ to be the maximum of $\Delta\left(r_{1}, r_{2}\right)$, where $r_{1}$ and $r_{2}$ are Dehn filling slopes of some simple manifold $M$ such that $M\left(r_{i}\right)$ is of type $X_{i}$. These numbers $\Delta\left(X_{1}, X_{2}\right)$ are now known in all ten cases; see [GW2] for more details.

Except when $\left(X_{1}, X_{2}\right)=(A, A),(A, T)$ or $(T, T)$, it is also known that $\Delta\left(X_{1}, X_{2}\right)$ is realized by infinitely many simple manifolds $M$; see [EW]. On the other hand, $\Delta(T, T)=8$, and there are exactly two simple manifolds $M$ admitting toroidal fillings $M\left(r_{1}\right), M\left(r_{2}\right)$ with $\Delta=\Delta\left(r_{1}, r_{2}\right)=8$, exactly one with $\Delta=7$, exactly one with $\Delta=6$, and infinitely many with $\Delta=5[\mathrm{Go}$. Similarly, $\Delta(A, T)=5$ [Go, GW1], and there is exactly one simple manifold $M$ having an annular filling $M\left(r_{1}\right)$ and a toroidal filling $M\left(r_{2}\right)$ with $\Delta=5$, exactly two with $\Delta=4$, and infinitely many with $\Delta=3$ [GW1]. In the present paper we complete the picture by dealing with the case $(A, A)$. In this case, $\Delta(A, A)=5$ [Go, GW1], and there are infinitely many simple manifolds $M$ admitting annular fillings $M\left(r_{1}\right), M\left(r_{2}\right)$ with $\Delta=3$ [GW1]. Here we show that there is exactly one such manifold $M$ with $\Delta=5$, and exactly two with $\Delta=4$. More precisely, we have the following theorem.

Theorem 1.1. Suppose $M$ is a compact, connected, orientable, irreducible, $\partial$ irreducible, anannular 3-manifold which admits two annular Dehn fillings $M\left(r_{1}\right)$, $M\left(r_{2}\right)$ with $\Delta=\Delta\left(r_{1}, r_{2}\right) \geq 4$. Then one of the following holds.

(1) $M$ is the exterior of the Whitehead link, and $\Delta=4$.

(2) $M$ is the exterior of the 2-bridge link associated to the rational number $3 / 10$, and $\Delta=4$.

(3) $M$ is the exterior of the $(-2,3,8)$ pretzel link, and $\Delta=5$.

The three manifolds listed in the theorem are the exteriors of the links in $S^{3}$ shown in Figure 1.1.

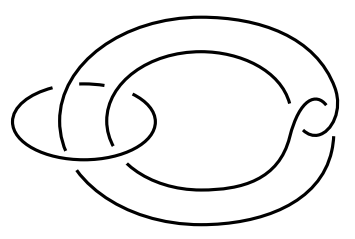

(1)

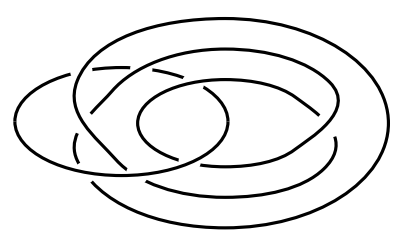

(2)

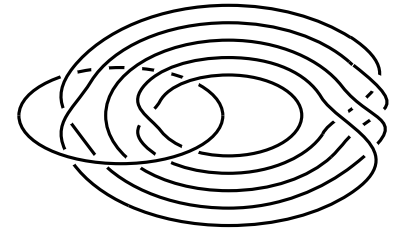

(3)

Figure 1.1

That each of these link exteriors does have a pair of annular fillings with $\Delta=4$, 
4 and 5 respectively is proved in [GW1]. The fillings in question are also toroidal [GW1], so in fact these are exactly the same manifolds which admit an annular and a toroidal filling with $\Delta \geq 4$ [GW1, Theorem 1.1]. Using [GW1, Theorem 1.1], Qiu has independently proved Theorem 1.1 in the special case where $M$ is the exterior of a knot in a solid torus [Q].

According to the proof of [GW1, Theorem 7.5], the annular fillings on the three manifolds listed in Theorem 1.1 are non Seifert fibered graph manifolds. If $M$ admits some Seifert fibered surgery, then $\partial M$ consists of tori, in which case $M$ is hyperbolic if and only if it is simple. Hence the following corollary is an immediate consequence of Theorem 1.1.

Corollary 1.2. Suppose $M$ is a compact orientable hyperbolic 3-manifold with at least two torus boundary components, and suppose $M\left(r_{1}\right), M\left(r_{2}\right)$ are Seifert fibered manifolds. Then $\Delta\left(r_{1}, r_{2}\right) \leq 3$.

The condition that $M$ has at least two boundary components cannot be removed. For example, if $M$ is the figure 8 knot complement, then $M(3)$ and $M(-3)$ are Seifert fibered, and $\Delta(-3,3)=6$. It is not known whether the bound 3 in the corollary is the best possible.

The proof of Theorem 1.1 proceeds as follows. For $\alpha=1,2$, let $A_{\alpha}$ be an essential annulus in $M\left(r_{\alpha}\right)$, meeting the Dehn filling solid torus $J_{\alpha}$ in $n_{\alpha}$ meridian disks, with $n_{\alpha}$ minimal over all choices of $A_{\alpha}$. This gives rise to a punctured annulus $F_{\alpha}=A_{\alpha} \cap M$ in $M$, such that the boundary components of $F_{\alpha}$ which lie on $T_{0}$ have slope $r_{\alpha}, \alpha=1,2$. The arcs of intersection of $F_{1}$ and $F_{2}$ then define labeled graphs $G_{\alpha}$ in $A_{\alpha}$ with $n_{\alpha}$ vertices, $\alpha=1,2$. We assume that $\Delta=\Delta\left(r_{1}, r_{2}\right)=4$ or 5 , and show by a detailed analysis that there are only three such pairs of graphs, corresponding to the three examples listed in the theorem.

The paper is organized as follows. In Section 2 we give some definitions and establish some basic properties of the graphs $G_{\alpha}$. In Section 3 we show that any graph in an annulus with no trivial loops or parallel edges must satisfy one of four possibilities; if the reduced graph $\widehat{G}_{\alpha}$ is of the fourth type we say that $G_{\alpha}$ is special. Section 4 is devoted to showing that if one of the graphs $G_{1}, G_{2}$ is special then they both are, and (up to relabeling) $n_{1}=1, n_{2}=2$. Section 5 considers the generic case, $n_{1}, n_{2}>2$. This is shown to be impossible, by eliminating in turn the first three possibilities of Section 3 for the reduced graphs $\widehat{G}_{\alpha}$. Section 6 shows that the case $n_{1}=2, n_{2}>2$ is also impossible. In Section 7 we show that if $G_{1}$ and $G_{2}$ are special, so $n_{1}=1$ and $n_{2}=2$, then there is exactly one possible pair $G_{1}, G_{2}$, with $\Delta=4$, corresponding to case (1) of Theorem 1.1. Finally in Section 8 , we show that if $G_{1}, G_{2}$ are not special and $n_{1}, n_{2} \leq 2$, then there are exactly two possible pairs $G_{1}, G_{2}$, one with $\Delta=4, n_{1}=n_{2}=2$, and one with $\Delta=5$, $n_{1}=n_{2}=2$, corresponding to cases (2) and (3) of Theorem 1.1.

We would like to thank the referee for his/her careful reading and helpful comments. 


\section{$\S 2$. Preliminary Lemmas}

Throughout this paper, we will always assume that $M$ is a compact, connected, irreducible, $\partial$-irreducible, anannular 3-manifold, with a torus boundary component $T_{0}$. We use $\alpha, \beta$ to denote the numbers 1 or 2 , with the convention that if they both appear, then $\{\alpha, \beta\}=\{1,2\}$. Let $r_{1}, r_{2}$ be slopes on $T_{0}$ such that $M\left(r_{1}\right), M\left(r_{2}\right)$ are annular, and let $A_{\alpha}$ be an essential annulus in $M\left(r_{\alpha}\right)$ such that $n_{\alpha}$, the number of components of intersection of $A_{\alpha}$ with the Dehn filling solid torus $J_{\alpha}$, is minimal among all essential annuli in $M\left(r_{\alpha}\right), \alpha=1,2$. Denote by $F_{\alpha}$ the punctured annulus $A_{\alpha} \cap M$. Denote by $\Delta=\Delta\left(r_{1}, r_{2}\right)$ the minimal geometric intersection number between $r_{1}$ and $r_{2}$. By [Go, Theorem 1.3] we have $\Delta \leq 5$. Throughout this paper, we will always assume $\Delta=4$ or 5 , unless otherwise stated.

Minimizing the number of components of $F_{1} \cap F_{2}$ by an isotopy, we may assume that $F_{1} \cap F_{2}$ consists of arcs and circles which are essential on both $F_{\alpha}$. Let $u_{1}, \ldots, u_{n_{\alpha}}$ be the disks that are the components of $A_{\alpha} \cap J_{\alpha}$, labeled successively when traveling along $J_{\alpha}$. Similarly let $v_{1}, \ldots, v_{n_{\beta}}$ be the disks in $A_{\beta} \cap J_{\beta}$. Let $G_{\alpha}$ be the graph on $A_{\alpha}$ with the $u_{i}$ 's as (fat) vertices, and the arc components of $F_{1} \cap F_{2}$ with at least one endpoint on $T_{0}$ as edges. Note that we do not regard an edge endpoint on the boundary of the annulus as a vertex, so we are abusing terminology somewhat in that our graphs may have edge endpoints that do not lie on vertices. The minimality of the number of components in $F_{1} \cap F_{2}$ and the minimality of $n_{\alpha}$ imply that $G_{\alpha}$ has no trivial loops, and that each disk face of $G_{\alpha}$ in $A_{\alpha}$ has interior disjoint from $F_{\beta}$.

An edge $e$ of a graph $G$ on an annulus $A$ is a boundary edge if it has one endpoint on the boundary of $A$, otherwise it is an interior edge. A vertex $v$ of $G$ is a boundary vertex if it is incident to a boundary edge, otherwise it is an interior vertex. Similarly, a face of $G$ is a boundary face if it contains a boundary edge.

If $e$ is an edge of $G_{\alpha}$ with an endpoint $x$ on a fat vertex $u_{i}$, then $x$ is labeled $j$ if $x$ is in $\partial u_{i} \cap \partial v_{j}$. When going around the boundary of a vertex in $G_{\alpha}$, the labels of the edge endpoints appear as $1,2, \ldots, n_{\beta}$ repeated $\Delta$ times.

An edge $e$ at a vertex $u_{i}$ of $G_{\alpha}$ is called a $j$-edge at $u_{i}$ if it has an endpoint at $u_{i}$ labeled $j$. Dually, a $j$-edge at $u_{i}$ is also an $i$-edge at $v_{j}$ in $G_{\beta}$. We say that $e$ is an $(i, k)$-edge if it has labels $i$ and $k$ at its two endpoints.

Each vertex of $G_{\alpha}$ is given a sign according to whether $J_{\alpha}$ passes $A_{\alpha}$ from the positive side or negative side at this vertex. Two vertices of $G_{\alpha}$ are parallel if they have the same sign, otherwise they are antiparallel. Note that if $A_{\alpha}$ is a separating surface in $M\left(r_{\alpha}\right)$, then $n_{\alpha}$ is even, and $u_{i}, u_{j}$ are parallel if and only if $i, j$ have the same parity. An interior edge of $G_{\alpha}$ is a positive edge if it connects parallel vertices. Otherwise it is a negative edge. We use $\operatorname{val}(v, G)$ to denote the valency of a vertex $v$ in a graph $G$.

By considering each family of parallel edges of $G_{\alpha}$ as a single edge $E$, we get the reduced graph $\widehat{G}_{\alpha}$ on $A_{\alpha}$. It has the same vertices as $G_{\alpha}$. Denote by $|E|$ the number of edges in $G_{\alpha}$ represented by $E$. 
A cycle in $G_{\alpha}$ consisting of positive edges is a Scharlemann cycle if it bounds a disk with interior disjoint from the graph, and all the edges in the cycle have the same pair of labels $(i, i+1)$ at their two endpoints. $\left(i+1=1\right.$ if $i=n_{\beta}$.) The pair $(i, i+1)$ is called the label pair of the Scharlemann cycle. In particular, a pair of adjacent parallel positive edges with the same label pair is a Scharlemann cycle. The boundary of the disk $D$ bounded by a Scharlemann cycle consists of edges of the Scharlemann cycle and some arcs on the annulus $C_{i}$ on $T_{0}$ between $\partial v_{i}$ and $\partial v_{i+1}$. When $n_{\beta}=2$, the two annuli $C_{1}$ and $C_{2}$ are still distinct, allowing one to differentiate between a $(1,2)$-Scharlemann cycle and a $(2,1)$-Scharlemann cycle. A pair of edges $\left\{e_{1}, e_{2}\right\}$ is an extended Scharlemann cycle if there is a Scharlemann cycle $\left\{e_{1}^{\prime}, e_{2}^{\prime}\right\}$ such that $e_{i}$ is parallel and adjacent to $e_{i}^{\prime}$.

A subgraph $G^{\prime}$ of a graph $G$ on a surface $F$ is essential if it is not contained in a disk in $F$.

Lemma 2.1. (1) (The Parity Rule) An edge e is a positive edge in $G_{1}$ if and only if it is a negative edge in $G_{2}$.

(2) A pair of edges cannot be parallel on both $G_{1}$ and $G_{2}$.

(3) If $G_{\alpha}$ has a set of $n_{\beta}$ parallel negative edges, then on $G_{\beta}$ they form mutually disjoint essential cycles of equal length.

(4) If $G_{\alpha}$ has a Scharlemann cycle, then $A_{\beta}$ is separating, and $n_{\beta}$ is even. Moreover, the edges of the Scharlemann cycle and the vertices at their endpoints form an essential subgraph of $G_{\beta}$.

(5) $G_{\alpha}$ contains no extended Scharlemann cycle.

Proof. See [GW1, Lemma 2.2], except for (2) in the case that the pair of edges $e_{1}, e_{2}$ are boundary edges. If $e_{1}, e_{2}$ are boundary edges parallel on both $G_{1}, G_{2}$, then they cut off bands $B_{1}, B_{2}$ on the punctured annuli $F_{1}, F_{2}$, which can be glued together to get an annulus in the manifold $M$, which intersects the Dehn filling torus $T_{0}$ in an essential circle. This contradicts the assumption that $M$ is $\partial$-irreducible and anannular.

Let $E$ be an edge of $\widehat{G}_{\alpha}$ representing $n_{\beta}$ parallel negative edges on $G_{\alpha}$, connecting $u_{i}$ to $u_{j}$. Then $E$ defines a permutation $\varphi:\left\{1, \ldots, n_{\beta}\right\} \rightarrow\left\{1, \ldots, n_{\beta}\right\}$, such that an edge $e$ in $E$ has label $k$ at $u_{i}$ if and only if it has label $\varphi(k)$ at $u_{j}$. Call $\varphi$ the permutation associated to $E$. Because of the ambiguity in the order of $u_{i}, u_{j}$, the permutation is only well defined up to inverse. An E-orbit is an orbit of $\varphi$. Such an orbit determines a cycle in $G_{\beta}$ consisting of the edges of $E$ with endpoint labels in this orbit, called the cycle of this orbit. Note that all the vertices in a cycle are parallel. Topologically each such cycle is a circle. Lemma 2.1(3) says that these circles are mutually disjoint, mutually parallel, essential circles on the annulus $A_{\beta}$.

Lemma 2.2. (1) Any two Scharlemann cycles on $G_{\alpha}$ have the same label pair. 
(2) If $E$ is a positive edge in $\widehat{G}_{\alpha}$, then $|E| \leq n_{\beta} / 2+1$. Moreover, if $|E|=$ $n_{\beta} / 2+1$, then the corresponding edges of $G_{\alpha}$ contain a Scharlemann cycle.

(3) Any family of parallel interior edges in $G_{\alpha}$ contains at most $n_{\beta}$ edges.

Proof. See [GW1, Lemma 2.5].

Lemma 2.3. (1) If some vertex of $G_{\alpha}$ has more than $n_{\beta}$ negative edge endpoints, then $G_{\beta}$ contains a Scharlemann cycle.

(2) No vertex of $G_{\alpha}$ has more than $2 n_{\beta}$ negative edge endpoints.

Proof. For any label $i$ of $G_{\beta}$, let $G_{\beta}^{+}(i)$ be the subgraph of $G_{\beta}$ consisting of all vertices of $G_{\beta}$ and all positive $i$-edges of $G_{\beta}$. The edges of $G_{\beta}^{+}(i)$ correspond to the negative edges of $G_{\alpha}$ incident to the vertex $u_{i}$. Let the number of such edges be $k$. Then if $f$ denotes the sum of the Euler characteristics of the faces of $G_{\beta}^{+}(i)$, we have

$$
0=\chi\left(A_{\beta}\right)=n_{\beta}-k+f .
$$

Therefore, if $k>n_{\beta}, G_{\beta}^{+}(i)$ has a disk face $D$. Then there is a Scharlemann cycle of $G_{\beta}$ in $D$ by [HM, Proposition 5.1]. This proves (1).

To prove (2), assume $k>2 n_{\beta}$. Then by the above we have $f=k-n_{\beta}>n_{\beta}$, so $G_{\beta}^{+}(i)$ has more than $n_{\beta}$ disk faces, and by [HM, Proposition 5.1] each such face contains a Scharlemann cycle of $G_{\beta}$. Hence $G_{\beta}$ contains $s>n_{\beta}$ Scharlemann cycles, all on the same label pair, say $(1,2)$, by Lemma 2.2(1). Define a graph $H$ in $A_{\beta}$ as follows; see [GL, Proof of Theorem 2.3]. The vertices of $H$ consist of the vertices of $G_{\beta}$, together with a vertex $v_{D}$ in the interior of each disk face of $G_{\beta}$ bounded by a Scharlemann cycle. The edges of $H$ are defined by joining each vertex $v_{D}$, within $D$, to the vertices of $G_{\beta}$ in $\partial D$. Thus $H$ has $n_{\beta}+s$ vertices and at least $2 s$ edges. An Euler characteristic argument then shows that $H$ has a disk face $E$. This disk $E$ contains a 1-cycle of $G_{\beta}$ (see [CGLS, p. 279] for definition), and hence a Scharlemann cycle [CGLS, Lemma 2.6.2]. But this contradicts the fact that $E$ is a face of $H$, because by definition $H$ would have a vertex in the disk bounded by this Scharlemann cycle.

Let $P, Q$ be two edge endpoints on the boundary of a vertex $u$ in $G_{\alpha}$. Let $P_{0}=P, P_{1}, \ldots, P_{k-1}, P_{k}=Q$ be the edge endpoints encountered when traveling along $\partial u$ in the direction induced by the orientation of $u$. Then the distance from $P$ to $Q$ (at the vertex $u$ ) is defined as $\rho_{u}(P, Q)=k$. Notice that since the valency of $u$ is $\Delta n_{\beta}$, we have $\rho_{u}(Q, P)=\Delta n_{\beta}-\rho_{u}(P, Q)$. If $e_{1}, e_{2}$ is a pair of edges, each having a single endpoint $P_{i}$ on the vertex $u$ in $G_{\alpha}$, then define $\rho_{u}\left(e_{1}, e_{2}\right)=\rho_{u}\left(P_{1}, P_{2}\right)$.

A pair of edges $e_{1}, e_{2}$ connecting two vertices $u, v$ in $G_{\alpha}$ is an equidistant pair if $\rho_{u}\left(e_{1}, e_{2}\right)=\rho_{v}\left(e_{2}, e_{1}\right)$. In particular, one can check that if $e_{1}, e_{2}$ are a pair of parallel edges connecting a pair of parallel vertices in $G_{\alpha}$, then $e_{1}, e_{2}$ is an equidistant pair in $G_{\alpha}$. 
Lemma 2.4. (The Equidistance Lemma.) Let $e_{1}, e_{2}$ be a pair of edges connecting the same vertices on $G_{1}$ and the same vertices on $G_{2}$. Then $e_{1}, e_{2}$ is an equidistant pair in $G_{1}$ if and only if it is an equidistant pair in $G_{2}$.

Proof. See [GW1, Lemma 2.8].

Given two slopes $r_{1}, r_{2}$ on the torus $T_{0}$, let $l$ be a curve intersecting $r_{1}$ at a single point. Choosing $l$ and the orientations of the curves properly, we may assume that homologically $r_{2}=q r_{1}+\Delta l$, where $1 \leq q<\Delta / 2$. The number $q$ is called the jumping number of $r_{1}, r_{2}$. Note that if $\Delta=4$ then $q=1$, and if $\Delta=5$ then $q=1$ or 2 .

Lemma 2.5. (1) If the jumping number $q=1$, in particular if $\Delta=4$, then a pair of $j$-edges at a vertex $u_{i}$ in $G_{\alpha}$ are adjacent among all the $j$-edges if and only if on $G_{\beta}$ they are also adjacent at $v_{j}$ among all $i$-edges.

(2) If $q=2$, then a pair of $j$-edges at a vertex $u_{i}$ in $G_{\alpha}$ are adjacent among all $j$-edges if and only if on $G_{\alpha}$ they are not adjacent among all the $i$-edges at $v_{j}$.

Proof. This is essentially [GW1, Lemma 2.10]. It was shown that if $P_{1}, \ldots, P_{\Delta}$ are the consecutive $j$-edge endpoints at $u_{i}$, then on $\partial v_{j}$ they appear in the order $P_{q}, P_{2 q}, \ldots, P_{\Delta q}$, hence the result follows.

A graph $G$ on an annulus $A$ is special if every vertex has at least two nonparallel boundary edges. Note that $G$ is special if and only if the corresponding reduced graph $\widehat{G}$ is special.

Lemma 2.6. (1) If $G$ is special then every vertex has exactly two boundary edges in $\widehat{G}$, going to distinct boundary components of $A$.

(2) If $G_{\alpha}$ has $2 n_{\beta}$ parallel boundary edges, then $G_{\beta}$ is special. $G_{\alpha}$ cannot have more than $2 n_{\beta}$ parallel boundary edges.

(3) If some edge $E$ of $\widehat{G}_{\alpha}$ represents $n_{\beta}$ negative edges, and if $G_{\alpha}$ has some positive edges, then $G_{\alpha}$ has at most $n_{\beta}$ parallel boundary edges, and each vertex of $\widehat{G}_{\beta}$ has at most one boundary edge.

Proof. (1) Otherwise there would be a pair of edges of $\widehat{G}$ at some vertex $v$ going to the same boundary component of $A$. By looking at an outermost such pair one can see that some vertex $u$ of $\widehat{G}$ has a single boundary edge in $\widehat{G}$, contradicting the definition of a special graph.

(2) If $G_{\alpha}$ has $2 n_{\beta}$ parallel boundary edges, then for any label $i$ it has two parallel $i$-edges. Since no two edges are parallel on both graphs, these two edges are non-parallel on $G_{\beta}$, hence $G_{\beta}$ is special. If $G_{\alpha}$ has more than $2 n_{\beta}$ parallel boundary edges, then there is a label $i$ such that $G_{\alpha}$ has three parallel boundary $i$-edges. Since by (1) the vertex $v_{i}$ in $\widehat{G}_{\beta}$ has only two boundary edges, two of 
these edges would be parallel on both graphs, contradicting Lemma 2.1(2).

(3) Since $G_{\alpha}$ has some positive edges, the vertices of $G_{\beta}$ cannot all be parallel, so there are at least two $E$-orbits, which form parallel essential cycles on $G_{\beta}$. Hence all boundary edges at a vertex of $G_{\beta}$ must be parallel to each other. If $G_{\alpha}$ has more than $n_{\beta}$ parallel boundary edges then two of them would be parallel on both graphs, contradicting Lemma 2.1(2).

Lemma 2.7. Suppose all vertices of $\widehat{G}_{\alpha}$ are boundary vertices, and suppose there are two boundary edges $E_{1}, E_{2}$ of $\widehat{G}_{\alpha}$ incident to the same vertex $v$ and going to the same boundary component of $A_{\alpha}$. Then $\widehat{G}_{\alpha}$ has a vertex $v^{\prime}$ of valency at most 3 which is incident to a single boundary edge, and $G_{\beta}$ is special.

Proof. Let $D$ be the disk on $A_{\alpha}$ cut off by $E_{1} \cup E_{2}$. Since $E_{1}, E_{2}$ are nonparallel, $D$ contains a vertex $v_{1} \neq v$, hence by adding an edge if necessary we may assume that there is an edge incident to $v$ other than $E_{1}, E_{2}$. Let $\widetilde{D}$ be the double of $D$ along $E_{1} \cup E_{2}$, and let $\widetilde{G}$ be the double of $\widehat{G}_{\alpha} \cap D$. Then each vertex of $\widetilde{G}$ has a boundary edge. By [CGLS, Lemma 2.6.5] $\widetilde{G}$ has a vertex $v^{\prime}$ of valency at most 3 and incident to at most one boundary edge. Since $v$ has valency at least 4 in $\widetilde{G}, v^{\prime} \neq v$. Hence $\operatorname{val}\left(v^{\prime}, \widehat{G}_{\alpha}\right)=\operatorname{val}\left(v^{\prime}, \widetilde{G}\right) \leq 3$. By Lemma $2.2(3)$ each interior edge of $\widetilde{G}_{\alpha}$ represents at most $n_{\beta}$ edges. Since $\Delta \geq 4$, this implies that the unique boundary edge at $v^{\prime}$ represents at least $2 n_{\beta}$ edges. By Lemma 2.6(2) in this case $G_{\beta}$ is special.

\section{$\S 3$. Reduced graphs on annuli}

By a reduced graph on a surface we mean one with no trivial loops or parallel edges; in other words, no faces of the graph are monogons or bigons.

Definition 3.1. Let $G$ be a reduced graph on an annulus $A$. Then $G$ is said to be triangular if

(i) every vertex has at most one boundary edge;

(ii) every interior vertex has valency 6 ;

(iii) every boundary vertex has valency 5 ;

(iv) every face of $G$ is a disk with three edges.

We remark that the only properties of a triangular graph that we will use are (i), (iii), and the fact that the graph has at least one boundary vertex (which follows from (iv)).

Proposition 3.1. Let $G$ be a reduced graph in an annulus $A$. Then either

(1) $G$ contains an interior vertex of valency at most 5 ; or

(2) $G$ contains a boundary vertex of valency at most 4 with exactly one boundary 
edge; or

(3) $G$ is triangular; or

(4) $G$ is special.

Proof. Let $G_{1}$ be a graph obtained from $G$ by adding extra edges so as to make each face of $G_{1}$ a disk with three edges. In particular, in $G_{1}$, each boundary face has three edges, and if some vertex $v$ has two boundary edges $e_{1}, e_{2}$, then $v$ has an edge on each side of $e_{1} \cup e_{2}$, so it has valency at least 4 .

Let $G_{2}$ be the union of $G_{1}$ and $\partial A$, with the obvious graph structure. Thus the points of $G_{1} \cap \partial A$ are now considered vertices, and the segments of $\partial A$ cut by these vertices are considered edges of $G_{2}$. Note that val $\left(v, G_{2}\right)=3$ for all vertices $v$ on $\partial A$, and each boundary face now has four edges. Let $G_{3}$ be obtained from $G_{2}$ by adding a diagonal edge in each boundary face of $G_{2}$, all sloping in the same direction; in other words, no two edges added have a common vertex on $\partial A$. We have $\operatorname{val}\left(v, G_{3}\right)=4$ if $v \in \partial A$. One can see that if we remove all edges and vertices on $\partial A$ then we get a graph that is obtained from $G_{1}$ by adding an extra copy of each boundary edge. Hence if $\operatorname{val}\left(v, G_{1}\right)=p$ and $v$ has $q$ boundary edges in $G_{1}$, then $\operatorname{val}\left(v, G_{3}\right)=p+q$. In particular, if $v$ has two boundary edges in $G_{1}$, then its valency in $G_{3}$ is at least $4+2=6$. Each face of $G_{3}$ is now a triangle.

The double of $A \underset{\widetilde{G}}{\text { along }} \partial A$ is a torus $T$, and the corresponding double of $G_{3}$ is a reduced graph $\widetilde{G}_{3}$ on $T$ with triangular faces. By an Euler characteristic argument, one can show that the number of edges in $\widetilde{G}_{3}$ is three times the number of vertices of $\widetilde{G}_{3}$. Thus either (i) some vertex $v$ of $\widetilde{G}_{3}$ has valency at most 5 , or (ii) all vertices of $\widetilde{G}_{3}$ have valency 6 . All vertices on $\partial A$ have valency 4 in $G_{3}$, hence valency 6 in $\widetilde{G}_{3}$, and we have shown that if $v$ has two boundary edges in $G_{1}$ then it has valency at least 6 in $G_{3}$; therefore (i) implies that either $v$ is an interior vertex of $G$ with valency at most 5 , or it is a boundary vertex of $G$ with valency at most $5-q \leq 4$ and incident to at most one boundary edge, so the graph is of type (1) or (2) in the proposition. Hence we may assume that all vertices of $G_{3}$ in the interior of $A$ have valency 6 .

If no vertex of $G_{1}$ has two boundary edges then each boundary vertex of $G_{1}$ has valency $6-q=6-1=5$. Since each interior vertex of $G_{1}$ has valency 6 , it follows that $G_{1}$ is triangular. Since $G$ is a subgraph of $G_{1}$ with the same vertices, either $G=G_{1}$ and hence $G$ is of type (3), or $G$ has a vertex $v$ with $\operatorname{val}(v, G)<\operatorname{val}\left(v, G_{1}\right)$, in which case $G$ is of type (1) or (2).

Now assume some vertex $v$ of $G_{1}$ has two boundary edges $e_{1}, e_{2}$ going to different boundary components. Then the valency of $v$ in $G_{1}$ is at most $6-2=4$. Since each face of $G_{1}$ has three edges, there is exactly one interior edge $e^{\prime}$ on each side of $e_{1} \cup e_{2}$. Let $v^{\prime}$ be the other endpoint of $e^{\prime}$. Since each face has three edges, $v^{\prime}$ must also have two boundary edges going to different boundary components of $A$. Repeating this process, we see that $G_{1}$ is a special graph such that each vertex has valency 4 . Since $G$ is a subgraph of $G_{1}$, either it is special, hence of type (4), or it has a vertex of valency at most 3 and incident to at most one boundary edge, 
in which case it is of type (1) or (2).

Finally, assume $G_{1}$ has a vertex $v$ which has two boundary edges going to the same boundary component. Then they cut off a disk $D$ from the annulus, which we may assume to be outermost. However, arguing as in the previous paragraph, we see that the vertex on the other end of an edge $e^{\prime}$ in $D$ incident to $v$ must have two boundary edges, which is a contradiction. Therefore this case does not happen.

\section{$\S 4$. Special graphs}

Recall that a graph $G$ on an annulus $A$ is special if every vertex has two nonparallel boundary edges. By Lemma 2.6(1) this implies that every vertex of $G$ has exactly

two boundary edges in $\widehat{G}$, going to different boundary components of $A$.

To simplify notation, denote $n_{\beta}$ by $n$.

Lemma 4.1. If $G_{\alpha}$ is a special graph, then $G_{\beta}$ is also special.

Proof. First notice that since each vertex $u_{i}$ of $G_{\alpha}$ is incident to at most two families of interior edges and each such family contains at most $n$ edges (Lemma $2.2(3))$, there are at most two interior $j$-edges at $u_{i}$ for any $j$. Hence there are at least $\Delta-2$ boundary $j$-edges at $u_{i}$. Since this is true for all $i, j$, we see that each vertex $v_{j}$ of $G_{\beta}$ has at least $2 n_{\alpha}\left(3 n_{\alpha}\right.$ if $\left.\Delta=5\right)$ boundary edges. Since each parallel family contains at most $2 n_{\alpha}$ edges (Lemma 2.6(2)), the lemma follows immediately when $\Delta=5$.

Now assume $\Delta=4$, and assume $G_{\beta}$ is not special. Then it has a vertex $v_{i}$ such that all boundary edges are parallel. By Lemma 2.6(2) and the above, $v_{i}$ has exactly $2 n_{\alpha}$ boundary edges, all parallel to each other. In particular, there are only two boundary 1 -edges $e_{1}, e_{1}^{\prime}$ at $v_{i}$. Dually this means that $e_{1}, e_{1}^{\prime}$ are the only boundary $i$-edges at $u_{1}$. Since they are parallel on $G_{\beta}$, they cannot be parallel on $G_{\alpha}$, so they belong to different families of boundary edges. Since these two edges are adjacent among all 1-edges at $v_{i}$, by Lemma 2.5(1) they must also be adjacent among all $i$-edges at $u_{1}$. This implies that the two interior $i$-edges at $u_{1}$ are on the same side of $e_{1} \cup e_{1}^{\prime}$, so they belong to the same edge $E$ in $\widehat{G}_{\alpha}$ because there is only one interior edge of $\widehat{G}_{\alpha}$ on each side of $e_{1} \cup e_{1}^{\prime}$. Since by Lemma $2.2(3) E$ contains at most $n$ edges, this is impossible.

In the remainder of this section we will assume that both $G_{1}$ and $G_{2}$ are special.

The sign of a vertex $u$ in $G_{\alpha}$ induces an orientation on $\partial u$, called its preferred orientation. Thus the preferred orientations of the $\partial u$ 's are all in the same direction on $T_{0}$. Let $e_{1}, e_{2}$ be a pair of adjacent boundary edges at some vertex $u$ of $G_{\alpha}$. When traveling on $\partial u$ along the preferred orientation, the labels at the endpoints of $e_{1}, e_{2}$ appear as $i, i+1$ for some $i(i+1=1$ if $i=n)$. They cut off a band $B$ on 
the surface $F_{\alpha}$, called an $i$-band at $u$ (of $G_{\alpha}$ ). Note that the label $i$ is determined by the pair $e_{1}, e_{2}$ even if $n=2$. The edge labeled $i$ at $u$ is called the initial edge of $B$, the other the terminal edge. Two $i$-bands of $G_{\alpha}$ are of different types if their initial edges are nonparallel on $G_{\beta}$; otherwise they are of the same type.

If $e_{1}, \ldots, e_{k}$ are all the edges of a parallel family $E$ at a vertex $u$, appearing in this order when traveling along the preferred orientation of $\partial u$, then $e_{k}$ is called the ending edge of $E$, and the label of $e_{k}$ at $u$ is called the ending label of $E$. Note that if a boundary $i$-edge $e$ is not an ending edge, then it is the initial edge of an $i$-band.

Lemma 4.2. There is a label $i$ such that all $i$-bands of $G_{\alpha}$ are of the same type.

Proof. Assuming otherwise, then there are two $i$-bands $B_{i}^{1}, B_{i}^{2}$ of different types for each $i$. Since the graph $G_{\beta}$ is special, there are only two families of parallel boundary edges for each vertex $v_{i}$ in $G_{\beta}$, so each family contains the initial edge of some $B_{i}^{j}$. Therefore, the terminal edge of each $B_{i}^{j}$ is parallel to the initial edge of some $B_{i+1}^{k}$, so there is a band $D_{i}^{j}$ on $F_{\beta}$ connecting these two edges. Note that $D_{i}^{j}$ degenerates to a single edge if these two edges coincide.

Consider the 2-complex $Q=\cup\left(B_{i}^{j} \cup D_{i}^{j}\right)$. Then $Q \cap T_{0}=\cup\left(e_{i}^{j} \cup d_{i}^{j}\right)$ is a graph $G$ on $T_{0}$, where $e_{i}^{j}=B_{i}^{j} \cap T_{0}$ and $d_{i}^{j}=D_{i}^{j} \cap T_{0}$. We have $Q \cong G \times I$. Shrinking each $d_{i}^{j}$ to a point, and orienting $e_{i}^{j}$ so that its endpoint is on $d_{i}^{j}$, we get an oriented graph $G^{\prime}$ in which each vertex $d_{i}^{j}$ is the tail of some edge $e_{i+1}^{k}$. Hence $G^{\prime}$ contains an embedded oriented cycle. The corresponding cycle $C$ in $G$ is then an embedded loop in $T_{0}$. Let $\gamma$ be a parallel copy of some boundary component of $F_{\beta}$ on $T_{0}$, intersecting some $e_{i}^{j}$ in $C$ transversely at a single point. The definition of $B_{i}^{j}$ and the orientation of $e_{i}^{j}$ implies that $C$ intersects $\gamma$ always in the same direction; hence $C$ is an essential curve. Thus $A=C \times I \subset Q$ is an annulus properly embedded in $M$ intersecting $T_{0}$ in the essential curve $C$, which contradicts the assumption that $M$ is $\partial$-irreducible and anannular.

Lemma 4.3. Each family of boundary edges in $G_{\alpha}$ contains at least $n$ edges.

Proof. Let $E_{1}, \ldots, E_{4}$ be the four edges of $\widehat{G}_{\alpha}$ at $u_{1}$, with $E_{1}, E_{3}$ the boundary edges. If $\left|E_{1}\right|<n$ then there is a label $i$ which does not appear at the endpoints of edges in $E_{1}$. If $\Delta=5$ then we would have $\left|E_{3}\right|=5 n-\left|E_{1}\right|-\left|E_{2}\right|-\left|E_{4}\right| \geq 2 n+1$, contradicting Lemma 2.6(2). Hence $\Delta=4$. Since $\left|E_{3}\right| \leq 2 n, E_{3}$ contains at most two $i$-edges, so each of $E_{2}, E_{4}$ contains one $i$-edge. Let $e_{1}, e_{2}$ be the $i$-edges of $E_{2}, E_{4}$ at $u_{1}$, and let $e_{3}, e_{4}$ be the $i$-edges of $E_{3}$. Since $e_{3}, e_{4}$ are adjacent $i$-edges at $u_{1}$, by Lemma 2.5(1) they are adjacent 1-edges at $v_{i}$. On $G_{\beta}$ the two edges $e_{3}, e_{4}$ belong to different families of boundary edges at $v_{i}$, because they cannot be parallel on both graphs. Therefore the two edges $e_{1}, e_{2}$ belong to the same family of interior edges. Since they both have label 1 at $v_{i}$, this would imply that the 
interior family containing them has at least $n_{\alpha}+1$ edges, contradicting Lemma $2.2(3)$.

Lemma 4.4. The jumping number $q=1$.

Proof. This is automatically true if $\Delta=4$. Hence assume $\Delta=5$. First assume that there is a vertex $u_{j}$ of $G_{\alpha}$ which has two interior $i$-edges $e_{1}, e_{2}$ for some $i$. Since each interior family contains at most $n$ edges, $e_{1}, e_{2}$ are nonparallel on $G_{\alpha}$. By Lemma 4.3 each boundary family contains an $i$-edge, hence $e_{1}, e_{2}$ are non adjacent among the $i$-edges at $u_{j}$. Dually on $G_{\beta}$ these are $j$-edges at the vertex $v_{i}$. For the same reason, they are non adjacent among all $j$-edges at $v_{i}$. Therefore by Lemma 2.5 the jumping number $q=1$.

Now assume that $u_{j}$ has at most one interior $i$-edge for all $i$. Then it has at most $n$ interior edge endpoints. On the other hand, since each boundary family contains at most $2 n$ edges and the valency of $u_{j}$ is $\Delta n=5 n$, we see that it cannot have less than $n$ interior edge endpoints; therefore it has exactly $n$ interior edge endpoints, and each boundary family contains exactly $2 n$ edges. If $u_{j}$ has two interior families, so each family contains less than $n$ edges, then the two boundary families have different ending labels. In this case for each label $i$ there are three $i$-bands, which cannot all be of the same type because each boundary family of $v_{i}$ has at most two $j$-edges. This contradicts Lemma 4.2. Therefore $u_{j}$ has only one family of interior edges, which contains $n$ edges. For the same reason, each vertex of $G_{\beta}$ has only one family of interior edges, containing $n_{\alpha}$ edges. By the parity rule one of these families is negative, and by Lemma 2.1(3) they form cycles on the other graph, so each vertex of that graph would then have two families of interior edges, contradicting the above conclusion.

Lemma 4.5. Suppose all $i$-bands at a vertex $u_{j}$ of $G_{\alpha}$ are of the same type. Then

(1) there are $n$ parallel interior edges at $u_{j}$, and

(2) each family of $n$ parallel interior edges at $u_{j}$ has $i$ as its ending label.

Proof. Let $E_{1}, \ldots, E_{4}$ be the edges at $u_{j}$ of $\widehat{G}_{1}$, appearing in this order around $\partial u_{j}$ along its preferred orientation, with $E_{1}, E_{3}$ the boundary edges. Let $e_{1}, \ldots, e_{4}$ be four $i$-edges at $u_{j}$, appearing successively along the preferred orientation of $\partial u_{j}$.

First assume that all $e_{i}$ are boundary edges. Then we may assume that $e_{1}, e_{2} \in$ $E_{1}$, and $e_{3}, e_{4} \in E_{3}$. Thus $e_{1}, e_{3}$ are not ending edges, so they are initial edges of some $i$-bands $B_{1}, B_{3}$. Since the jumping number $q=1$ (Lemma 4.4), and since $e_{1}, e_{3}$ are non adjacent among $i$-edges at $u_{j}$, by Lemma 2.5 they are non adjacent among $j$-edges at $v_{i}$ in $G_{\beta}$, hence they are non parallel boundary edges on $G_{\beta}$. Therefore $B_{1}, B_{3}$ are of different type.

Now assume that $E_{2}$ contains an $i$-edge $e_{2}$, say. Since each of $E_{1}, E_{3}$ contains at least $n$ edges, we must have $e_{1} \in E_{1}$ and $e_{3} \in E_{3}$. Assume that either $e_{2}$ is not the ending edge of $E_{2}$ or $\left|E_{2}\right|<n$. Then $e_{1}$ is not an ending edge of $E_{1}$, and there 
is an $i$-band $B_{1}$ with $e_{1}$ as the initial edge. If $e_{3}$ is not an ending edge either, then there is an $i$-band $B_{3}$ with $e_{3}$ as initial edge. For the same reason as above, $B_{1}, B_{3}$ are of different type, and we are done. So assume that $e_{3}$ is the ending edge of $E_{3}$. Now we must have $\left|E_{4}\right|<n$ as otherwise $E_{4}$ would have $n$ edges and have the $i$-edge $e_{4}$ as its ending edge, contradicting the assumption. Hence $e_{4}$ is in $E_{1}$, and so there is an $i$-band with $e_{4}$ as an initial edge. Since $e_{1}, e_{4}$ are parallel on $G_{\alpha}$, they are nonparallel on $G_{\beta}$, so again $B_{1}, B_{4}$ are of different type. This proves (1). To prove (2), notice that if $\left|E_{2}\right|=n$ but $e_{2}$ is not the ending edge, then $e_{1}, e_{3}$ are not ending edges of $E_{1}, E_{3}$, so from the above the two $i$-bands $B_{1}, B_{3}$ are of different type.

Lemma 4.6. Suppose $n>2$. Then each positive edge of $\widehat{G}_{\alpha}$ represents at most $n / 2$ edges.

Proof. When $n>2$, the special graph $\widehat{G}_{\beta}$ has at most one edge connecting any two vertices. If $G_{\alpha}$ has $n / 2+1$ parallel positive edges, then it has a Scharlemann cycle $e_{1} \cup e_{2}$ with label pair $(1,2)$, say. So the two edges $e_{1}, e_{2}$ would be parallel on $G_{\beta}$, contradicting Lemma 2.1(4).

Proposition 4.7. If $G_{\alpha}$ is special then up to relabeling we have $n_{1}=1, n_{2}=2$, and $G_{1}$ has exactly two interior edges.

Proof. First assume $n_{\alpha} \geq 2$ for $\alpha=1,2$. By Lemma 4.2 for each graph $G_{\alpha}$ there is a label $i$ such that all $i$-bands of $G_{\alpha}$ are of the same type. Let $u_{j}$ be a vertex of $G_{\alpha}$. By Lemma 4.5(1), it has a set of $n$ parallel interior edges $E$ with $i$ as its ending label at $u_{j}$. Let $u_{k}$ be the vertex on the other endpoint of $E$, then by Lemma 4.5(2), $E$ also has ending label $i$ at $u_{k}$. If $E$ is negative, then the ending edge $e$ of $E$ at $u_{j}$ is the same as that at $u_{k}$, so $e$ would have the same label $i$ on its two endpoints, and hence is a loop on $G_{\beta}$. Since $n \geq 2$ and $G_{\beta}$ is special, this is absurd. If $E$ is positive, then the two ending edges would give rise to two negative edges at $v_{i}$ in $G_{\beta}$, which must be nonparallel because they cannot be parallel on both graphs. Thus both families of interior edges at $v_{i}$ are negative. Replacing $u_{j}$ by $v_{i}$ in the above argument, we get a contradiction because now $E$ must be negative. Therefore up to relabeling we must have $n_{1}=1$.

By Lemma 4.5(1) the only vertex $u_{1}$ of $G_{1}$ has $n_{2}$ parallel interior edges, which by the parity rule must be negative edges on $G_{2}$, hence $n_{2} \geq 2$. If $n_{2}>2$, then by Lemma $4.6 G_{1}$ has at most $n_{2} / 2$ interior edges, which is a contradiction. Hence the result follows. 


\section{$\S 5$. The generic case}

In this section except for Lemma 5.1, we assume $n_{\alpha}, n_{\beta}>2$. By Proposition 4.7, $G_{\alpha}$ and $G_{\beta}$ are not special. Again denote $n_{\beta}$ by $n$.

Lemma 5.1. Suppose $n_{\alpha} \geq 2, n>2$, and suppose $\widehat{G}_{\alpha}$ has a negative edge $E$ with $|E|=n$, and a positive edge $E^{\prime}$ with $\left|E^{\prime}\right|=n / 2+1$. Let $(1,2)$ be the label pair of the Scharlemann cycle in $E^{\prime}$. Then

(1) $G_{\beta}$ has at most $n / 2$ boundary vertices;

(2) when $n=4$, the two vertices $v_{3}, v_{4}$ of $G_{\beta}$ cannot both be boundary vertices; and

(3) when $n=4, G_{\alpha}$ cannot have both a $(1,4)$-edge and a $(2,3)$-edge.

Proof. Let $k$ be the number of $E$-orbits. Since $E^{\prime}$ contains more than $n / 2$ edges, hence contains a Scharlemann cycle, the annulus $A_{\beta}$ is separating, so $G_{\beta}$ has the same number of positive and negative vertices. Each $E$-orbit contains the same number $(n / k)$ of vertices, all of the same sign, so the number of orbits containing positive vertices is the same as the number of those containing negative ones, and hence $k$ must be even. Recall that each $E$-orbit forms an essential cycle on $G_{\beta}$, so only the vertices on the two cycles adjacent to the two boundary components of $A_{\beta}$ could be boundary vertices. Hence the number of boundary vertices is at most $2(n / k)$, and since $k$ is even, (1) follows unless $k=2$.

Assume $k=2$. Let $C_{1}, C_{2}$ be the two cycles of $E$-orbits on $G_{\beta}$, and let $e_{1}, e_{2}$ be the edges of the Scharlemann cycle in $E^{\prime}$. By Lemma 2.1(4) $e_{1} \cup e_{2}$ is an essential cycle on $G_{\beta}$. The two vertices $v_{1}, v_{2}$ of $e_{1} \cup e_{2}$ are on different $C_{1}, C_{2}$ because they are antiparallel, so the cycle $e_{1} \cup e_{2}$ lies between $C_{1}$ and $C_{2}$, separating the vertex $v_{3}$ on the first orbit from the vertex $v_{n}$ on the second. On the other hand, since $E^{\prime}$ contains more than two edges, there is an edge adjacent to the Scharlemann cycle which is a $(3, n)$-edge, so on $G_{\beta}$ there would be an edge connecting $v_{3}$ to $v_{n}$. This is a contradiction, showing that $k=2$ is impossible. In particular, this proves (1).

Now assume $n=4$. Since we have shown that $k$ is even and $k \neq 2$, we must have $k=4$. In this case each vertex $v_{i}$ of $G_{\beta}$ has an essential loop $C_{i}$ coming from the $n$ parallel negative edges in $G_{\alpha}$. These loops and their vertices form essential circles on $A_{\beta}$ which are parallel to each other. As above, there is an edge in $E^{\prime}$ which connects $v_{3}$ to $v_{4}$. Hence the circles $C_{3}$ and $C_{4}$ are adjacent to each other, so $v_{3}, v_{4}$ cannot both be boundary vertices. This proves (2). Since the edges in the Scharlemann cycle connect $v_{1}$ to $v_{2}, C_{1}$ is adjacent to $C_{2}$. Thus either $C_{3}$ separates $v_{4}$ from $v_{1}, v_{2}$, so there is no edge connecting $v_{4}$ to $v_{1}$, or $C_{4}$ separates $v_{3}$ from $v_{1}, v_{2}$, so there is no edge connecting $v_{3}$ to $v_{2}$. This proves (3).

Lemma 5.2. Suppose $E_{1}, \ldots, E_{5}$ are the edges of $\widehat{G}_{\alpha}$ at a vertex $u$ of valency 5 . If $E_{1}, E_{2}, E_{3}$ are positive, and $E_{4}$ is an interior edge, then $\left|E_{5}\right|>n$; in particular, $E_{5}$ is a boundary edge. 
Proof. Assume $\left|E_{5}\right| \leq n$. By Lemma 2.2(2) we have $\left|E_{i}\right| \leq n / 2+1$ for $i \leq 3$, and by Lemma 2.2(3) $\left|E_{4}\right| \leq n$. Since $n>2$, we must have $\Delta=4$, and

$$
4 n=\Delta n=\left|E_{1}\right|+\cdots+\left|E_{5}\right| \leq 3\left(\frac{n}{2}+1\right)+2 n=\frac{7}{2} n+3,
$$

which implies that $n \leq 6$. Moreover, $n$ must be even, otherwise by Lemmas $2.2(2)$ and 2.1(4) we would have $\left|E_{i}\right| \leq n / 2$ for $i=1,2,3$, hence $4 n \leq 3(n / 2)+2 n$, which is absurd.

If $n=6$, then all the above inequalities are equalities. In particular, $\Delta=4$, $\left|E_{4}\right|=\left|E_{5}\right|=6$, and $\left|E_{i}\right|=4$ for $i=1,2,3$, so each of $E_{1}, E_{2}, E_{3}$ contains a Scharlemann cycle, and by Lemma 2.2(1) they all have the same label pair, say $(1,2)$. But since $\left|E_{4}\right|=\left|E_{5}\right|=n$, these labels also appear in $E_{4}$ and $E_{5}$. Thus the label 1 appears 5 times, contradicting the fact that $\Delta=4$.

Now assume $n=4$. If each of $E_{1}, E_{2}, E_{3}$ contains a Scharlemann cycle with label pair $(1,2)$, say, (in particular, if $\left|E_{i}\right|=3$ for $i=1,2,3$ ), then again the labels $\{1,2\}$ appear three times among the endpoints of $E_{1} \cup E_{2} \cup E_{3}$ at $u$. Also, since $E_{4} \cup E_{5}$ has at least $16-3 \times 3=7$ edge endpoints at $u$, one of the labels $\{1,2\}$ appears at least twice among the endpoints of $E_{4} \cup E_{5}$ at $u$, so it appears 5 times at $u$, contradicting the fact that $\Delta=4$. Hence we may assume that $\left|E_{1}\right|=\left|E_{2}\right|=3$, $\left|E_{3}\right|=2, E_{3}$ contains no Scharlemann cycle, and $\left|E_{4}\right|=\left|E_{5}\right|=4$. Since the two edges of $E_{3}$ have labels 3,4 at $u$, they must have label sets $\{1,4\}$ and $\{2,3\}$. Since $\left|E_{4}\right|=4$, the edges in $E_{4}$ are negative. This contradicts Lemma 5.1(3), completing the proof of the lemma.

Lemma 5.3. $\widehat{G}_{\alpha}$ has no interior vertex of valency at most 5 .

Proof. Let $E_{1}, \ldots, E_{5}$ be the edges of $\widehat{G}_{\alpha}$ incident to $u$. Since all these edges are interior edges, by Lemma 5.2 they can have at most two positive edges, say $E_{1}, E_{2}$. By Lemma $2.3(2), u$ has at most $2 n$ negative edges in $G_{\alpha}$, hence $E_{1} \cup E_{2}$ represents at least $2 n$ positive edges. By Lemma $2.2(2)$ we have $2 n \leq 2(n / 2+1)$, which contradicts the assumption that $n \geq 3$.

Lemma 5.4. $\widehat{G}_{\alpha}$ cannot have a boundary vertex $u$ of valency at most 4 with a single boundary edge.

Proof. Let $E_{0}$ be the boundary edge, and $E_{1}, E_{2}, E_{3}$ the interior edges of $\widehat{G}_{\alpha}$ at $u$. By Lemma 2.6(2) and Proposition 4.7 we have $\left|E_{0}\right|<2 n$. By Lemma 2.3(2), $u$ can have at most $2 n$ negative edges in $G_{\alpha}$, so one of the interior edges, say $E_{1}$, must be positive, and by Lemma $2.2(2)\left|E_{1}\right| \leq n / 2+1<n$. Since each of $E_{2}, E_{3}$ represents at most $n$ edges, we have $\left|E_{0}\right|>n$.

We claim that either $G_{\alpha}$ or $G_{\beta}$ contains a Scharlemann cycle. If $u$ has more than $n$ negative edges, then by Lemma 2.3(1) $G_{\beta}$ contains a Scharlemann cycle. So assume $u$ has at most $n$ negative edges. Since $u$ has less than $2 n$ boundary 
edges, it must have more than $n$ positive edges. If at most two of the $E_{1}, E_{2}, E_{3}$ are positive, then one of them represents more than $n / 2$ positive edges; if all the three interior edges at $u$ are positive, then since they represent more than $2 n$ edges, again one of them represents more than $n / 2$ edges. In either case these parallel edges contain a Scharlemann cycle. This completes the proof of the claim.

Now $\left|E_{0}\right|>n$ implies that some vertex of $G_{\beta}$ has two nonparallel boundary edges. In particular, $\widehat{G}_{\beta}$ cannot be triangular. It follows from Lemma 5.3 and Proposition 3.1 that $\widehat{G}_{\beta}$ must also have a boundary vertex $v$ of valency at most 4 with a single boundary edge. Since one of $G_{\alpha}$ and $G_{\beta}$ has a Scharlemann cycle, by considering $v$ instead of $u$ if necessary, we may assume without loss of generality that $G_{\alpha}$ contains a Scharlemann cycle with label pair $(1,2)$.

We claim that $\left|E_{0}\right| \leq n+2$. Otherwise each vertex of $G_{\beta}$ has a boundary edge, and some vertex $v_{i}$ other than $v_{1}, v_{2}$ has two such edges $e_{1}, e_{2}$. Since the edges of the Scharlemann cycle form an essential subgraph of $G_{\beta}$ (Lemma 2.1(4)), separating the two boundary components of $A_{\beta}$, the edges $e_{1}, e_{2}$ must go to the same boundary component. Applying Lemma 2.7, we see that $G_{\alpha}$ is special, a contradiction.

Since $\left|E_{0}\right|>n$ and $u$ has some positive edges, by Lemma 2.6(3) the graph $G_{\alpha}$ cannot have $n$ parallel negative edges. Thus if $k$ of the $E_{1}, E_{2}, E_{3}$ are positive, then

$$
4 n \leq(n+2)+k\left(\frac{n}{2}+1\right)+(3-k)(n-1)=\left(4-\frac{k}{2}\right) n+(2 k-1)
$$

which implies that $n<4$. But since $G_{\alpha}$ contains a Scharlemann cycle, $n$ is even. This contradicts the assumption that $n>2$.

Lemma 5.5. If both $\widehat{G}_{1}, \widehat{G}_{2}$ are triangular, then each boundary vertex has exactly two positive and two negative edges in $\widehat{G}_{\alpha}$.

Proof. Let $E_{0}, \ldots, E_{4}$ be the edges of $\widehat{G}_{\alpha}$ at a boundary vertex $v$, with $E_{0}$ the boundary edge. Since $\widehat{G}_{\beta}$ is also triangular, $E_{0}$ represents at most $n$ edges. Therefore by Lemma 5.2 at most two of the $E_{i}$ are positive. On the other hand, by Lemma 2.3(2) $v$ has at most $2 n$ negative edges, hence at least $n$ positive edges. Since each $E_{i}$ represents at most $n / 2+1<n$ positive edges, $v$ must have two positive edges in $\widehat{G}_{\alpha}$.

Lemma 5.6. Suppose both $\widehat{G}_{1}, \widehat{G}_{2}$ are triangular. Then all vertices of $G_{1}, G_{2}$ are boundary vertices.

Proof. Let $E_{0}, \ldots, E_{4}$ be the edges of $\widehat{G}_{\alpha}$ at a boundary vertex $v$, with $E_{0}$ the boundary edge. By Lemma 5.5 we may assume that $E_{1}, E_{2}$ are negative edges, and $E_{3}, E_{4}$ are positive edges.

Suppose $G_{\beta}$ has some interior vertices. Then $\left|E_{0}\right|<n$. Since $v$ has at most $2 n$ negative edges, it has more than $n$ positive edges, so $E_{3} \cup E_{4}$ contains a Scharle- 
mann cycle with label pair $(1,2)$, say, and $n$ is even. Also, one of $E_{1}, E_{2}$ must represent $n$ parallel edges, for otherwise $E_{0} \cup E_{1} \cup E_{2}$ would contain at most $3 n-3$ edges, so one of $E_{3}, E_{4}$ would contain at least $n / 2+2$ edges, contradicting Lemma $2.2(2)$. Now we can apply Lemma $5.1(1)$ and conclude that $G_{\beta}$ has at most $n / 2$ boundary vertices. Thus $\left|E_{0}\right| \leq n / 2$. We have the inequality

$$
4 n \leq\left|E_{0}\right|+\ldots+\left|E_{5}\right| \leq \frac{n}{2}+2 n+2\left(\frac{n}{2}+1\right) \leq \frac{7}{2} n+2 .
$$

Since $n$ is even, this implies $n=4,\left|E_{0}\right|=2,\left|E_{1}\right|=E_{2} \mid=4$, and $\left|E_{3}\right|=\left|E_{4}\right|=3$. Now each of $E_{3}, E_{4}$ contains a Scharlemann cycle on label pair $(1,2)$, so these labels appear 4 times among the interior edge endpoints at $v$. Thus the labels of $E_{0}$ must be 3,4. This contradicts Lemma 5.1(2).

Lemma 5.7. $\widehat{G}_{1}, \widehat{G}_{2}$ cannot both be triangular.

Proof. Assume $\widehat{G}_{1}, \widehat{G}_{2}$ are triangular. Then by Lemma 5.6 all vertices of $G_{1}, G_{2}$ are boundary vertices, and by Lemma 5.5 each vertex $v$ of $G_{\alpha}$ has exactly two positive edges and two negative edges in $\widehat{G}_{\alpha}$. Since a positive edge in $G_{\alpha}$ is a negative edge in $G_{\beta}$, it follows that either (i) some vertex $v$ of one of the graphs, say $G_{1}$, has more positive edge endpoints than negative ones, or (ii) all vertices of $G_{1}$ and $G_{2}$ have the same number of positive and negative edge endpoints.

In case (i), (writing $\left.n=n_{2}\right), v$ has at most $2(n / 2+1)=n+2$ positive edges, at most $n+1$ negative edges, and at most $n$ boundary edges. From the inequality

$$
4 n \leq(n+2)+(n+1)+n
$$

we see that $n \leq 3$. But if $n=3$ then $v$ has at most $2(n / 2)=n$ positive edges, at most $n-1$ negative edges, and at most $n$ boundary edges, which would lead to the contradiction that $4 n \leq n+(n-1)+n$.

In case (ii), any vertex $v$ of $G_{\alpha}$ has at most $n+2$ positive edges, the same number of negative edges, and at most $n$ boundary edges; so from $4 n \leq(n+2)+(n+2)+n$ we see that $n=4,|E|=3$ for all positive interior edges of $\widehat{G}_{\alpha}$, and $|E|=4$ for all boundary edges of $G_{\alpha}$. Each label appears three times among the interior edge endpoints at any vertex $v$ of $G_{\alpha}$, but since each of the two families of positive edges at $v$ contains a Scharlemann cycle, which must all have the same label pair $(1,2)$, it follows that these labels appear only once among the negative edge endpoints at $v$, so the label 3 appears twice among the negative edge endpoints at $v$. Since this is true for all vertices $v$ in $G_{\alpha}$, it means that the vertex $v_{3}$ on $G_{\beta}$ has $2 n_{\alpha}$ positive edge endpoints, and $n_{\alpha}$ negative ones, a contradiction.

Proposition 5.8. One of the graphs $G_{\alpha}$ has at most two vertices.

Proof. Assume $n_{1}, n_{2} \geq 3$. By Proposition $4.7, G_{\alpha}$ is not special, by Lemma 5.3 $\widehat{G}_{\alpha}$ does not have an interior vertex of valency at most 5 , and by Lemma 5.4 it 
cannot have a boundary vertex of valency at most 4 with a single boundary edge. Thus by Proposition 3.1 both $\widehat{G}_{1}, \widehat{G}_{2}$ are triangular, which contradicts Lemma 5.7.

\section{$\S$. Nonspecial graphs with $n_{1}=2$ and $n_{2}>2$}

Throughout this section we will assume that $G_{1}, G_{2}$ are not special graphs. We will show that the case $n_{1}=2$ and $n_{2}=n>2$ does not happen. Together with Propositions 4.7 and 5.8, this shows that $n_{\alpha}$ must be at most 2 for both $\alpha=1$ and 2.

Lemma 6.1. If $n_{1}=2$ then $\widehat{G}_{1}$ is a subgraph of that shown in Figure 6.1.

Proof. Since $\widehat{G}_{1}$ is not a special graph, one of the vertices $u_{1}, u_{2}$ has at most one boundary edge. If either $u_{1}$ or $u_{2}$ does not have a loop, then one can find a vertex $u$ of valency at most 3 in $\widehat{G}_{1}$, with at most one boundary edge. Since each interior edge represents at most $n$ edges, $u$ would have at least $2 n$ boundary edges, which would imply that $G_{2}$ is a special graph, a contradiction. Hence each vertex $u_{i}$ has a loop. It is now easy to see that $\widehat{G}_{1}$ must be a subgraph of that in Figure 6.1.

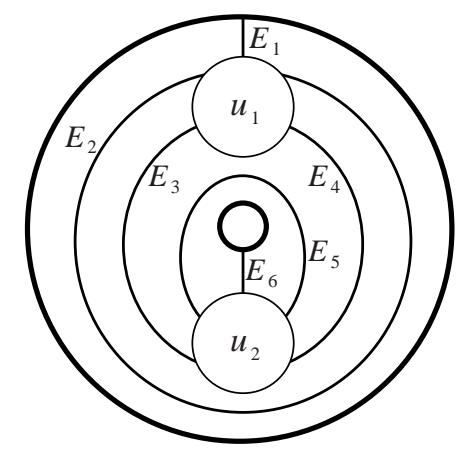

Figure 6.1

Label the edges of $\widehat{G}_{1}$ as in Figure 6.1. Denote by $m$ the number of non-loop interior edges of $G_{1}$, i.e. $m=\left|E_{3}\right|+\left|E_{4}\right|$.

Lemma 6.2. Suppose $n_{1}=2$, and $n>2$.

(1) Either $m=2 n$, or $m=2 n-2$ and $E_{2}$ contains a Scharlemann cycle.

(2) The two vertices of $G_{1}$ are antiparallel.

Proof. (1) If no label appears twice among the endpoints of edges in $E_{2}$, then from the labeling on $\partial u_{1}$ one can see that either $m \geq 2 n$ or $\left|E_{1}\right| \geq 2 n$. But the second 
possibility does not occur because then by Lemma 2.6(2) the graph $G_{2}$ would be special. Hence in this case we have $m \geq 2 n$. Since each of $E_{3}, E_{4}$ represents at most $n$ edges, we conclude that $m=2 n$.

Now assume that some label appears twice among the endpoints of edges in $E_{2}$. Then $E_{2}$ contains a Scharlemann cycle $e_{1}, e_{2}$, with label pair $(1,2)$, say. Since $n>2, E_{2}$ contains no extended Scharlemann cycle (Lemma 2.1(5)), so one of these two edges, say $e_{1}$, must be an outermost edge among those in $E_{2}$. Thus the endpoints of $e_{1}$ are either adjacent to those in $E_{3} \cup E_{4}$ or to those in $E_{1}$. In the first case, the label sequence of $E_{3} \cup E_{4}$ at $u_{1}$ is $3,4, \ldots, n$, so $m \equiv n-2 \bmod n$. If $m=2 n-2$ then we are done. If $m \neq 2 n-2$, then since $\left|E_{3}\right|,\left|E_{4}\right| \leq n$, we must have $m=n-2$. Thus $\left|E_{1}\right|=\Delta n-m-2\left|E_{2}\right| \geq 2 n$, which by Lemma 2.6(2) would imply that $G_{2}$ is special, a contradiction. Therefore $e_{1}$ must be adjacent to $E_{1}$. As above, we have either $\left|E_{1}\right|=2 n-2$, or $\left|E_{1}\right|=n-2$ and $m \geq 2 n$. In the second case we have $m=2 n$ because $\left|E_{3}\right|,\left|E_{4}\right| \leq n$. It remains to show that $\left|E_{1}\right|=2 n-2$ is impossible.

Assume $\left|E_{1}\right|=2 n-2$. Notice that this happens only if $E_{2}$ contains a Scharlemann cycle. Moreover, if $(1,2)$ is the label pair of the Scharlemann cycle then all labels other than 1,2 would appear twice among endpoints of edges in $E_{1}$. Thus on $G_{2}$ each vertex other than $v_{1}, v_{2}$ would have two boundary 1-edges. But since the edges in the Scharlemann cycle and the vertices $v_{1}, v_{2}$ form an essential subgraph of $G_{2}$, these two parallel 1-edges must go to the same boundary component of $A_{2}$. By looking at an outermost vertex one can see that there is a vertex $v_{i}$ with $i \neq 1,2$, at which the two boundary 1-edges are parallel, so they are parallel on both graphs, contradicting Lemma 2.1(2).

(2) If $u_{1}, u_{2}$ are parallel then $2 n-2 \leq\left|E_{3}\right|+\left|E_{4}\right| \leq 2(n / 2+1)$, implying that $n=4$ and $\left|E_{3}\right|=\left|E_{4}\right|=3$. In this case both $E_{3}, E_{4}$ contain Scharlemann cycles, and by Lemma 2.2(1) they must have the same label pair $(1,2)$ as the one in $E_{2}$. But since each of the labels 1,2 appears only once among the endpoints at $u_{1}$ of edges in $E_{3} \cup E_{4}$, this is impossible.

Lemma 6.3. Suppose $n_{1}=2$, and $n>2$. Then $G_{1}$ cannot have $2 n$ negative edges.

Proof. We must have $\left|E_{2}\right|>0$, otherwise $\left|E_{1}\right| \geq 2 n$, so $G_{2}$ would be special, contradicting our assumption. Assume that $G_{1}$ has $2 n$ negative edges. Then $\left|E_{3}\right|=\left|E_{4}\right|=n$, and by Lemma 2.6(3) we have $\left|E_{1}\right| \leq n$, hence $\left|E_{2}\right| \geq n / 2$. On the other hand, by Lemma $2.2(2)\left|E_{2}\right| \leq n / 2+1$. Hence $E_{2}$ contains either $n / 2$ or $n / 2+1$ edges. We want to show that $\left|E_{2}\right| \neq n / 2+1$. Assuming otherwise, then since $E_{3}$ contains $n$ parallel negative edges, by Lemma 5.1 the graph $G_{1}$ has at most $n / 2$ parallel boundary edges. On the other hand, we have $\left|E_{1}\right|=$ $\Delta n-m-2\left|E_{2}\right| \geq n-2$, and since $E_{2}$ contains a Scharlemann cycle with label pair $(1,2)$, say, $n$ is even. Therefore we must have $n=4$. Now in this case the labels of the edges in $E_{1}$ are 3,4, contradicting Lemma 5.1(2). 


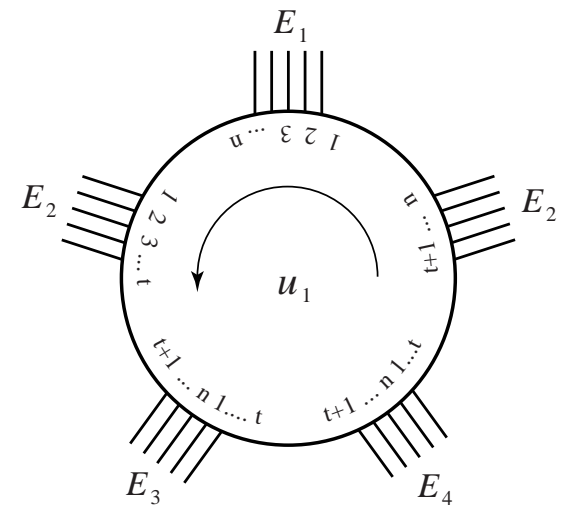

(a)

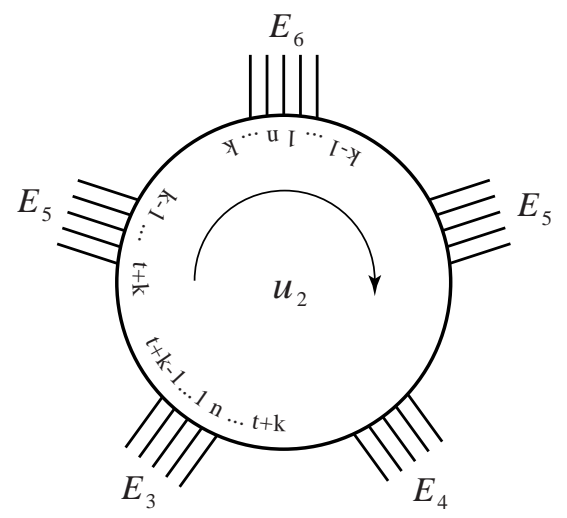

(b)

Figure 6.2

We have shown that $\left|E_{2}\right|=n / 2$, and $\left|E_{1}\right|=n$. For the same reason, we have $\left|E_{5}\right|=n / 2$ and $\left|E_{6}\right|=n$. Without loss of generality we may assume that $u_{1}$ is a positive vertex, $u_{2}$ is negative, and the edges of $E_{1}$ have label sequence $1, \ldots, n$ at $u_{1}$. See Figure 6.2. Let $t=n / 2$. Since $\left|E_{2}\right|=n / 2$, the label sequence of the endpoints at $u_{1}$ of the edges of $E_{3}$ is $t+1, \ldots, n, 1, \ldots, t$. There is a number $k$ such that the label sequence at the other end of $E_{3}$ is $t+k, t+k+1, \ldots, t+k-1$. The number $k \neq 1$, otherwise these edges would be loops in $G_{2}$, so $n>2$ would imply that some vertex of $G_{2}$ does not have a boundary edge, contradicting the fact that $\left|E_{1}\right|=n$. Now from Figure 6.2 we can see that the label sequence of $E_{6}$ is $k, \ldots, n, 1, \ldots, k-1$, hence the two edges $e_{3}, e_{4}$ in $E_{6}$ labeled $n$ and 1 respectively, are adjacent (because $k \neq 1$ ). Let $e_{1}, e_{2}$ be the edges of $E_{1}$ labeled 1 and $n$ respectively. By Lemma 2.6(3), each vertex of $G_{2}$ has at most one family of parallel boundary edges, so $e_{2}$ is parallel to $e_{3}$, and $e_{4}$ parallel to $e_{1}$ in $G_{2}$. Let $B\left(e_{1}, e_{2}\right)$ be the band on $F_{1}$ between $e_{1}$ and $e_{2}$, and let $B\left(e_{3}, e_{4}\right)$ be that between $e_{3}$ and $e_{4}$. Similarly, let $B\left(e_{2}, e_{3}\right)$ and $B\left(e_{4}, e_{1}\right)$ be the bands on $F_{2}$ between $e_{2}, e_{3}$ and $e_{4}, e_{1}$, respectively. Now we can form an annulus $A=B\left(e_{1}, e_{2}\right) \cup B\left(e_{2}, e_{3}\right) \cup B\left(e_{3}, e_{4}\right) \cup B\left(e_{4}, e_{1}\right)$ in the manifold $M$. Since the boundary curve $C$ of $A$ on $T_{0}$ intersects the circle $\partial v_{2}$ transversely at a single point (on the $\operatorname{arc} B\left(e_{1}, e_{2}\right) \cap \partial u_{1}$ ), it is an essential curve. This contradicts the fact that the manifold $M$ is $\partial$-irreducible and anannular.

Lemma 6.4. Suppose $n_{1}=2$, and $n>2$. Then $G_{1}$ cannot have exactly $2 n-2$ negative edges.

Proof. If $G_{1}$ has $2 n-2$ negative edges, then (up to symmetry) either $\left|E_{3}\right|=\left|E_{4}\right|=$ $n-1$, or $\left|E_{3}\right|=n$ and $\left|E_{4}\right|=n-2$. Looking at the labeling, one can see that 
the two loops of $E_{2}$ near $E_{3} \cup E_{4}$ form a Scharlemann cycle, with label pair $(1,2)$, say. If $\left|E_{3}\right|=n$ then by Lemma $2.6(3)$ we have $\left|E_{1}\right| \leq n$, hence $\left|E_{2}\right| \geq n / 2+1$. Now by Lemma $5.1(1)$ the graph $G_{2}$ has at most $n / 2$ boundary vertices, which contradicts the fact that $\left|E_{1}\right|=n$. Therefore we must have $\left|E_{3}\right|=\left|E_{4}\right|=n-1$.

For the same reason, the two loops in $E_{5}$ near $E_{3} \cup E_{4}$ form a Scharlemann cycle, which by Lemma 2.2(1) must have the same label pair $(1,2)$. Now we can see that $E_{3}$ has label sequence $3,4, \ldots, n, 1$, at $u_{1}$, and has label sequence $2,3, \ldots, n$ at $u_{2}$. However, in this case $E_{3}$ has only one orbit, containing all the labels, so all the vertices of $G_{2}$ are parallel to each other, hence all edges of $G_{1}$ are negative. But since $G_{1}$ contains some loops, this is a contradiction.

Proposition 6.5. If $M\left(r_{1}\right), M\left(r_{2}\right)$ are annular, and $\Delta \geq 4$, then $n_{\alpha} \leq 2$ for $\alpha=1,2$.

Proof. By Proposition 4.7 this is true if one of the $G_{\alpha}$ is special. By Proposition 5.8 one of the graphs, say $G_{1}$, has at most two vertices. Since the two possibilities in Lemma 6.2(1) have been ruled out by Lemmas 6.3 and 6.4, the case $n_{1} \leq 2$ and $n_{2}>2$ cannot happen.

\section{$\S$ 7. Special graphs with $n_{1}=1$ and $n_{2}=2$}

Proposition 7.1. If $G_{\alpha}$ is special, then $\Delta=4$, up to relabeling $n_{1}=1, n_{2}=2$, and the manifold $M$ is the exterior of the Whitehead link.

Proof. By Lemma 4.1, both graphs must be special. By Proposition 4.7, up to relabeling we must have $n_{1}=1, n_{2}=2$, and $G_{1}$ has exactly two interior edges $e_{1}, e_{2}$.

Assume $\Delta=5$. By Lemma 4.4 the jumping number $q=1$. There is a pair of adjacent boundary 1-edges $e_{1}, e_{2}$ at $v_{1}$ in $G_{2}$, which by Lemma 2.5(1) should also be adjacent at $u_{1}$ in $G_{1}$ among all 1-edges; but since the two families of boundary edges at $u_{1}$ are separated by two interior edges, $e_{1}, e_{2}$ must be in the same family, so they are parallel on both graphs, a contradiction. Therefore we must have $\Delta=4$.

Now the Whitehead link exterior $W$ does admit two annular Dehn fillings $W\left(r_{1}\right), W\left(r_{2}\right)$ with $\Delta\left(r_{1}, r_{2}\right)=4, n_{1}=1$, and $n_{2}=2$, see [GW1, Theorem 7.5]. It remains to show that the manifold satisfying these conditions is unique.

Each vertex of $G_{2}$ has two boundary edges, which are nonparallel because $G_{2}$ is special. Thus the graph $G_{2}$ must be as shown in Figure 7.1(b). Similarly, since $G_{1}$ is special it has two families of parallel boundary edges. The loops have different labels at their two endpoints, so each family of boundary edges of $G_{1}$ contains an even number of edges. Hence $G_{1}$ must be as shown in Figure 7.1(a).

Label the six edges of $G_{1}$ as in the figure. Orient $e_{3}, e_{4}$ so that on $G_{1}$ they 


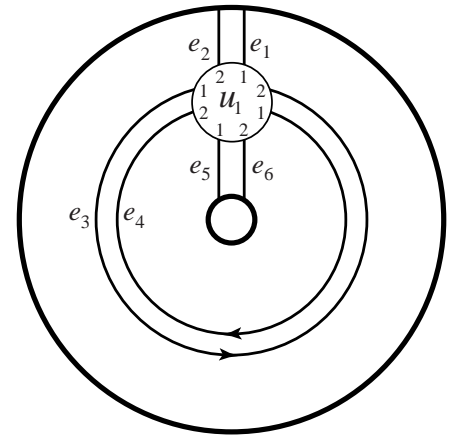

(a)

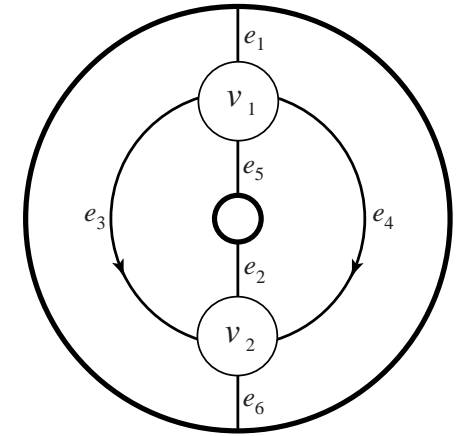

(b)

Figure 7.1

have label 1 at their tails. Up to symmetry we may assume that the edge $e_{1}$ on $G_{2}$ is as shown in Figure 7.1(b). The label 1 endpoints of edges $e_{1}, e_{3}, e_{5}, e_{4}$ appear successively on $\partial u_{1}$, hence by Lemma 2.5(1) they also appear in this order on $\partial v_{1}$ in $G_{2}$, so these edges must be as shown in the figure. Similarly by looking at the label 2 endpoints of $e_{2}, e_{4}, e_{6}, e_{3}$ one can determine the edges $e_{4}$ and $e_{6}$. Therefore up to symmetry the graphs $G_{\alpha}$ are exactly as shown in the figure. We need to show that these graphs uniquely determine the manifold $M$.

Recall that $F_{\alpha}$ denotes the punctured annulus $A_{\alpha} \cap M$. Let $X=N\left(F_{1} \cup T_{0}\right)$, and let $Y=N\left(F_{1} \cup F_{2} \cup T_{0}\right)$, where the regular neighborhoods are taken in $M$. The frontier of $X$ in $M$, i.e. $X \cap \overline{M-X}$, is a surface $F$, which is a four punctured sphere. Note that $Y$ is obtained from $X$ by adding regular neighborhoods of the faces of $G_{2}$. Each of the four faces of $G_{2}$ is a disk $D_{i}$ with $\partial D_{i}=c_{i} \cup c_{i}^{\prime}$, where $c_{i}^{\prime}$ is an $\operatorname{arc}$ on $\partial M$, and $c_{i}$ an $\operatorname{arc}$ on $F_{1} \cup T_{0}$. Let $\widetilde{c}_{i}$ be the $\operatorname{arc} D_{i} \cap F$. Then the frontier of $Y=X \cup\left(\cup N\left(D_{i}\right)\right)$ in $M$ is a properly embedded surface $F^{\prime}$, homeomorphic to the surface obtained by cutting $F$ along the $\operatorname{arcs} \widetilde{c}_{i}$. Thus $Y$ and $X$ are homeomorphic, but they are embedded in $M$ differently. Note that $Y$ is uniquely determined by the graphs $G_{1}$ and $G_{2}$.

It is easy to see that all the $\widetilde{c}_{i}$ are essential arcs on $F$. Since each boundary component of $F$ meets $\cup \widetilde{c}_{i}$ twice, after cutting along all these $\widetilde{c}_{i}$, the remnant, and hence $F^{\prime}$, consists of either two disks, or two disks and an annulus. In fact, by examining the graphs, one can see that $F^{\prime}$ indeed consists of two disks and an annulus. Since $M$ is irreducible and $\partial$-irreducible, the disk components of $F^{\prime}$ are boundary parallel. If the annular component $A$ of $F^{\prime}$ is incompressible in $M$ then $A$ is also boundary parallel because $M$ is anannular and irreducible, so $M$ would be homeomorphic to $Y$, which in turn is homeomorphic to $X$. Let $C$ be an essential curve on $T_{0}$ disjoint from $\partial F_{1}$. Then $C \times I$ in $T_{0} \times I$ would be an essential annulus in $X$, contradicting the fact that $M$ is anannular. Therefore $A$ must be compressible. Let $D$ be a compressing disk of $A$ in $M$. Then $D$ lies in 
either $Y$ or $M-\operatorname{Int} Y$. We show that the first case is impossible.

First notice that the surface $F_{1}$ cuts $X$ into a manifold $F \times I$, in which both $F$ and the two copies of $F_{1}$ are incompressible. By an innermost circle argument one can show that $F$ is incompressible in $X$. Under the homeomorphism $Y \cong X, A$ can be considered as a subsurface of $F$, hence $A$ is also incompressible unless the core of $A$ is a trivial curve on $F$. On the other hand, notice that $A$ is a component of $\partial Y-\operatorname{Int} F^{\prime \prime}$, where $F^{\prime \prime}=Y \cap\left(\partial M-T_{0}\right)$ is a neighborhood of $\partial A_{1} \cup \partial A_{2}$ on $\partial M$, which is connected. Since $\partial A \subset F^{\prime \prime}$, it follows that the core of $A$ is nonseparating on $\partial Y$, hence it is nontrivial on $F$. This completes the proof that $A$ is incompressible in $Y$.

Hence the compressing disk $D$ of $A$ lies in $M-\operatorname{Int} Y$. Let $M^{\prime}$ be the union of $Y$ and a regular neighborhood of $D$. Then the frontier of $M^{\prime}$ in $M$ is a set of disks, which must be boundary parallel because $M$ is irreducible and $\partial$-irreducible. Therefore $M^{\prime}$ is homeomorphic to $M$. It follows that $M$ is obtained from $Y$ by adding a 2-handle along the core of $A$, and hence is uniquely determined by the graphs $G_{1}$ and $G_{2}$.

\section{§. Nonspecial graphs with $n_{\alpha} \leq 2$}

First note that if $n_{\alpha}=1$ and $G_{\alpha}$ is not special, then the unique vertex of $G_{\alpha}$ has valency at most 3 in $\widehat{G}_{\alpha}$, and hence by Lemma $2.2(3) G_{\alpha}$ has at least $2 n_{\beta}$ parallel boundary edges. By Lemma 2.6(2) this implies that $G_{\beta}$, and therefore (by Lemma 4.1) $G_{\alpha}$, is special, a contradiction. Hence if $G_{1}, G_{2}$ are not special and $n_{1}, n_{2} \leq 2$, we must have $n_{1}=n_{2}=2$.

Lemma 8.1. Suppose that $n_{1}=n_{2}=2$ and $G_{1}, G_{2}$ are not special. Then for $\alpha=1,2$, the two vertices of $G_{\alpha}$ are antiparallel, $\widehat{G}_{\alpha}$ is a subgraph of the graph $\widehat{G}$ in Figure 6.1, and one of the following holds.

(i) $\Delta=4$, each interior edge of $\widehat{G}_{\alpha}$ represents two edges of $G_{\alpha}$, and $G_{\alpha}$ has no boundary edges.

(ii) $\Delta=5$, each edge of $\widehat{G}_{\alpha}$ represents two edges of $G_{\alpha}$, and the jumping number $q=2$.

Proof. By Lemma 6.1, $G_{\alpha}$ is a subgraph of the graph $\widehat{G}$ shown in Figure 6.1. Each vertex $v$ of $G_{\alpha}$ must have a loop, otherwise some vertex would have valency 3 in $\widehat{G}_{\alpha}$ with a single boundary edge, so by Lemma 2.6(2) $G_{\beta}$ would be special, contradicting the assumption. Since a loop in $G_{\alpha}$ is a non-loop negative edge of $G_{\beta}$, it follows that each graph $G_{\beta}$ has some negative edges, hence the two vertices of $G_{\beta}$ must be antiparallel, $\beta=1,2$. By Lemma 2.2(3) each interior edge of $\widehat{G}_{\alpha}$ represents at most two edges of $G_{\alpha}$. Similarly, each boundary edge of $\widehat{G}_{\alpha}$ also represents at most two edges of $G_{\alpha}$, by Lemma 2.1(2).

First assume $\Delta=4$. Notice that a vertex of $G_{\alpha}$ has either no boundary edge or 
two boundary edges, for if it has exactly one boundary edge then the loops based at that vertex would have the same label at their two endpoints, which contradicts the parity rule. Since two boundary edges at a vertex of $G_{\alpha}$ correspond to boundary edges at different vertices of $G_{\beta}$, it follows that either both vertices of $G_{\alpha}$ have two boundary edges, or they both have no boundary edges. The second possibility gives rise to conclusion (i) in the lemma.

Assume that each vertex of $G_{\alpha}$ has two boundary edges. Then there are a total of 6 interior edges in each graph. Note that an interior edge is a loop on $G_{\alpha}$ if and only if it is a non-loop on $G_{\beta}$ because of the parity rule, hence one of the graphs, say $G_{1}$, has at least three loops. Without loss of generality we may assume that there are two loops $e_{1}, e_{2}$ based at the vertex $u_{1}$. Consider their label 1 endpoints. Because there are two boundary edges at $u_{1}$, these two endpoints are non adjacent among all label 1 endpoints at $u_{1}$. Now look at the graph $G_{2}$. By Lemma 2.5(1) $e_{1}, e_{2}$ are non adjacent 1-edges at $v_{1}$ among all 1-edges. However, since they are non-loops in $G_{2}$, they are contained in the two adjacent families $E_{3}, E_{4}$ in Figure 6.1. Since $E_{3} \cup E_{4}$ contains a total of at most four edges, $e_{1}, e_{2}$ are adjacent among all 1-edges at $v_{1}$. This contradiction completes the proof of the lemma for the case $\Delta=4$.

Now assume $\Delta=5$. Since each vertex of $\widehat{G}$ has valency 5 , and since each edge of $\widehat{G}_{\alpha}$ represents at most two edges of $G_{\alpha}, \Delta=5$ implies that each edge of $\widehat{G}_{\alpha}$ represents exactly two edges. By the same argument as above one can show that the jumping number $q$ cannot be 1 , so we are in case (ii).

Lemma 8.2. There is a unique irreducible, $\partial$-irreducible, anannular manifold $M$ which admits two annular Dehn fillings $M\left(r_{1}\right), M\left(r_{2}\right)$ with $\Delta\left(r_{1}, r_{2}\right)=5$.

Proof. By Lemma 8.1, the graphs must be as shown in Figure 8.1. We first show that the edge correspondence and the labelings of the vertices are unique up to symmetry.

Reflecting the annuli vertically and changing their orientations if necessary, we may assume that the vertices $u_{1}, v_{1}$ are positive, and the labeling of edge endpoints at $\partial u_{1}, \partial v_{1}$ are as shown. Any non-loop edge has the same label on its two endpoints, because it is a loop edge on the other graph. Thus the labeling on $\partial u_{2}, \partial v_{2}$ is determined by that on $\partial u_{1}, \partial v_{1}$, respectively. Orient the edges so that a non-loop edge goes from $u_{1}$ to $u_{2}$ (resp. $v_{1}$ to $v_{2}$ ). Then dually the orientation of a loop edge must go from label 1 to label 2. Label the edges of $G_{1}$ as in Figure 8.1(a).

If $P_{1}, \ldots, P_{5}$ are the points of $u_{1} \cap v_{1}$, appearing in this order on $\partial u_{1}$ along its orientation, then since the jumping number $q=2$, they appear in the order $P_{1}, P_{3}, P_{5}, P_{2}, P_{4}$ on $\partial v_{1}$ either along or against the orientation of $\partial v_{1}$. In other words, along the orientation of $\partial v_{1}$ they either appear in this order, or in the order $P_{1}, P_{4}, P_{2}, P_{5}, P_{3}$. In the second case, write $\left(Q_{1}, Q_{2}, \ldots, Q_{5}\right)=\left(P_{1}, P_{4}, P_{2}, P_{5}, P_{3}\right)$; then $\left(P_{1}, \ldots, P_{5}\right)=\left(Q_{1}, Q_{3}, Q_{5}, Q_{2}, Q_{4}\right)$. Hence by interchanging the roles of $G_{1}$ 
and $G_{2}$ if necessary, we may assume that the points appear as $\left(P_{1}, P_{3}, P_{5}, P_{2}, P_{4}\right)$ on $\partial v_{1}$ along the orientation of $\partial v_{1}$.

Now we can see that the labeling of the edges on $G_{2}$ is completely determined by that of $G_{1}$ : The 1-edges at $u_{1}$ appear in the order $a, c, e, k, d$ in the positive direction, so at $v_{1}$ they appear in the order $a, e, d, c, k$, where $a$ is the unique boundary edge at $v_{1}$ labeled 1 . The order of the 2-edges at $u_{1}$ is $b, d, f, l, c$, so dually the 1-edges at $v_{2}$ are in the order $b, f, c, d, l$. Similarly by looking at $u_{2}$ one can determine the labeling of the remaining edges in $G_{2}$. See Figure 8.1(b).

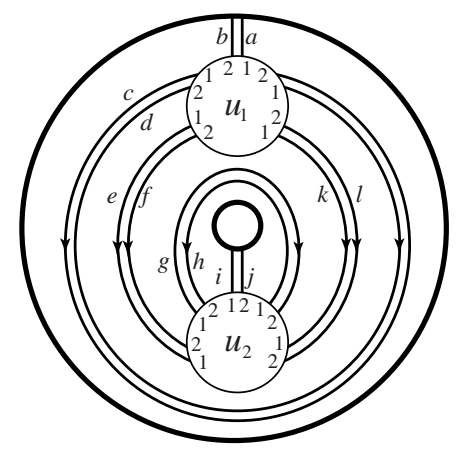

(a)

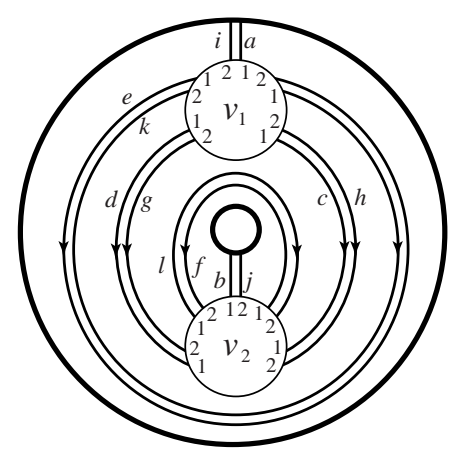

(b)

Figure 8.1

It remains to show that the manifold $M$ is uniquely determined by these graphs. As in the proof of Proposition 7.1, consider the submanifold $X=N\left(A_{1} \cup J_{1}\right)$ of $M\left(r_{1}\right)$. Since $J_{1}$ intersects $A_{1}$ in two meridian disks of opposite sign, the frontier $F$ of $X$ consists of two components $F_{b}, F_{w}$, each being a twice punctured torus, called the black surface and the white surface respectively. A face of $G_{2}$ is black or white according to whether it intersects the black surface or the white surface. Note that each face of $G_{2}$ intersects $F$ in a circle or an arc, so it is either black or white, but not both.

Let $D_{1}$ be a face of $G_{2}$ bounded by a pair of parallel loops, and let $D_{2}$ be the triangular interior face of $G_{2}$ adjacent to $D_{1}$. Since they have an edge in common, they are of different colors, so we may assume that $D_{1}$ is black and $D_{2}$ is white. The boundary of $D_{1}$ intersects a meridian of $J_{1}$ twice in the same direction, hence $\partial D_{1}$ is a nonseparating curve on $F_{b}$. After adding a neighborhood of $D_{1}$ to $X$, the black frontier is homeomorphic to the surface obtained by 2-surgery on $F_{b}$ along $\partial D_{1}$, hence is an annulus $A_{b}$. Since its boundary components are essential curves on $\partial M$, and since $M$ is $\partial$-irreducible, $A_{b}$ is incompressible in $M$, and hence is boundary parallel in $M$. Similarly, since the boundary of $D_{2}$ intersects a meridian of $J_{1}$ three times, $\partial D_{2}$ is a nonseparating curve on $F_{w}$, so after adding $N\left(D_{2}\right)$ the white frontier becomes an annulus $A_{w}$, which for the same 
reason must be boundary parallel in $M$. It follows that $M$ is homeomorphic to $N\left(F_{1} \cup T_{0} \cup D_{1} \cup D_{2}\right)$, where $F_{1}$ is the punctured annulus $A_{1} \cap M$. The boundary curves of $D_{i}$ are determined by the graphs, which have been determined (up to symmetry) as above. Hence the manifold $M$ is uniquely determined.

Lemma 8.3. There is a unique irreducible, $\partial$-irreducible, anannular manifold $M$ which admits two annular Dehn fillings $M\left(r_{1}\right), M\left(r_{2}\right)$ with $\Delta\left(r_{1}, r_{2}\right)=4$ and $n_{1}=n_{2}=2$.

Proof. The proof is similar to that of Lemma 8.2. In this case the jumping number is 1 , and one can show that up to symmetry the graphs must be as shown in Figure 8.2. The proof that $M$ is determined by the graphs is the same as in the proof of Lemma 8.2.

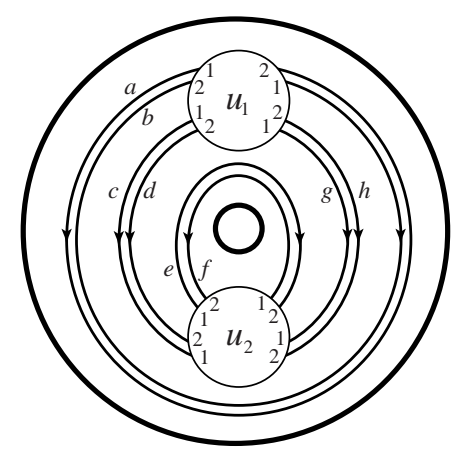

(a)

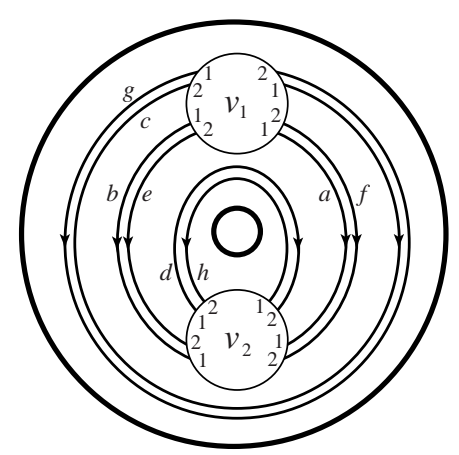

(b)

Figure 8.2

We now prove Theorem 1.1, which we restate here for the reader's convenience.

Theorem 1.1. Suppose $M$ is a compact, connected, orientable, irreducible, $\partial-$ irreducible, anannular 3-manifold which admits two annular Dehn fillings $M\left(r_{1}\right)$, $M\left(r_{2}\right)$ with $\Delta=\Delta\left(r_{1}, r_{2}\right) \geq 4$. Then one of the following holds.

(1) $M$ is the exterior of the Whitehead link, and $\Delta=4$.

(2) $M$ is the exterior of the 2-bridge link associated to the rational number $3 / 10$, and $\Delta=4$.

(3) $M$ is the exterior of the $(-2,3,8)$ pretzel link, and $\Delta=5$.

Proof. By Proposition 6.5, we must have $n_{\alpha} \leq 2$ for $\alpha=1,2$. If $G_{\alpha}$ is special, then by Proposition 7.1 the manifold $M$ is the exterior of the Whitehead link. If $G_{\alpha}$ is nonspecial, then by Lemma 8.1 the graphs $G_{\alpha}$ must be as in Figure 8.1 or 8.2, and by Lemmas 8.2 and 8.3 , in each case the manifold $M$ is uniquely determined by the 
graphs; hence there are at most three manifolds $M$ which may admit two annular Dehn fillings of distance at least 4 apart. On the other hand, it has been shown in [GW1, Theorem 7.5] that each of these manifolds admits two such fillings. Hence the result follows.

\section{References}

[CGLS] M. Culler, C. Gordon, J. Luecke and P. Shalen, Dehn surgery on knots, Annals Math. 125 (1987), 237-300.

[EW] M. Eudave-Muñoz and Y-Q. Wu, Nonhyperbolic Dehn fillings on hyperbolic 3-manifolds, Pac. J. Math. 190 (1999), 261-275.

[Go] C. Gordon, Boundary slopes of punctured tori in 3-manifolds, Trans. Amer. Math. Soc. 350 (1998), 1713-1790.

[GL] C. Gordon and J. Luecke, Reducible manifolds and Dehn surgery, Topology 35 (1996), 385-409.

[GW1] C. Gordon and Y.-Q. Wu, Toroidal and annular Dehn fillings, Proc. London Math. Soc. 78 (1999), 662-700.

[GW2] C. Gordon and Y.-Q. Wu, Annular and boundary reducing Dehn fillings, Topology 39 (2000), 531-548.

[HM] C. Hayashi and K. Motegi, Only single twists on unknots can produce composite knots, Trans. Amer. Math. Soc. 349 (1997), 4465-4479.

[Q] R. Qiu, Annular Dehn surgery on knots in a solid torus, Northeast. Math. J. 15 (1999), 130-132.

[Th1] W. Thurston, Three dimensional manifolds, Kleinian groups and hyperbolic geometry, Bull. Amer. Math. Soc. 6 (1982), 357-381.

[Th2] W. Thurston, The Geometry and Topology of 3-manifolds Princeton University, 1978.

Cameron McA. Gordon

Department of Mathematics

University of Texas at Austin

Austin, TX 78712

USA

e-mail: gordon@math.utexas.edu
Ying-Qing Wu

Department of Mathematics

University of Iowa

Iowa City, IA 52242

USA

e-mail:wu@math.uiowa.edu

(Received: February 20, 1999) 